Check for updates

Cite this: RSC Adv., 2017, 7, 48386

\title{
Hydrothermal synthesis of nitrogen and boron doped carbon quantum dots with yellow-green emission for sensing $\mathrm{Cr}(\mathrm{vI})$, anti-counterfeiting and cell imaging $\dagger$
}

\author{
Yongming Guo, (iD *a Yuzhi Chen, ${ }^{\text {bc }}$ Fengpu Cao, ${ }^{a}$ Lijuan Wang, ${ }^{a}$ Zhuo Wang (D) *bc \\ and Yumin Leng ${ }^{\mathrm{d}}$
}

Nitrogen and boron doped carbon quantum dots (NB-CQDs) with yellow-green emission were prepared via hydrothermal method using 2-hydroxyphenylboronic acid and ethylenediamine in aqueous solution. The as-prepared NB-CQDs exhibited bright yellow-green emission, excellent stability in aqueous solution with high ionic strength and good biocompatibility. We further explored the applications of NBCQDs for sensing metal ions in aqueous solution. We found that the NB-CQDs could be utilized to sense $\mathrm{Cr}_{2} \mathrm{O}_{7}{ }^{2-}$ in aqueous solution due to the $\mathrm{Cr}_{2} \mathrm{O}_{7}{ }^{2-}$-induced fluorescence quenching. In the sensing system, as low as $0.5 \mu \mathrm{M} \mathrm{Cr}_{2} \mathrm{O}_{7}{ }^{2-}$ can be successfully detected and a good linear relationship in the range of $0-250 \mu \mathrm{M}$ has been obtained. And the NB-CQDs can be also used as ink for writing on paper, revealing the NB-CQDs can be applied for anti-counterfeiting. Moreover, the NB-CQDs exhibit negligible cytotoxicity and good biocompatibility, which is favorable for bioimaging applications. And the NB-CQDs were successfully applied for fluorescence imaging in HeLa cells.

Received 3rd September 2017

Accepted 30th September 2017

DOI: 10.1039/c7ra09785a

rsc.li/rsc-advances easy fabrication, low-cost, excellent biocompatibility, good photostability, bright fluorescent properties, good water solubility, low cytotoxicity. ${ }^{4}$ Therefore, considerable efforts have been dedicated to preparation of various CQDs with different emission, and conspicuous progresses of these CQDs on diverse applications including bioimaging, biosensing, photocatalysis, and optoelectronic devices have been made., ${ }^{4,7-9}$

In recent years, heteroatoms-doped CQDs have become research hot spots because the incorporation of heteroatoms in the framework of the CQDs can improve or change the properties of these CQDs, such as optical, electrical and other properties, resulting into more promising applications. Huang's group has prepared nitrogen-doped CQDs (N-CQDs) from the hydrothermal treatment of citric acid and guanidine hydrochloride. And they found that nitrogen doping could improve the emission efficiency and favor excitation-independent emission. ${ }^{\mathbf{1 0}} \mathrm{Ma}$ and co-workers have synthesized N-CQDs from the ultrasonic reaction between glucose and ammonium hydroxide. And the $\mathrm{N}$ CQDs showed strong emission in the visible-to-near infrared range and upconversion photoluminescence properties. Surprisingly, the N-CQDs displayed good photocatalytic property for the photodegradation of methyl orange under visible light. ${ }^{11}$ Citric acid and polyethylenimine have been utilized to prepare blue-emitting N-CQDs, which can be employed for cell imaging. Interestingly, the N-CQDs can cross the blood-brain barrier in a concentration-dependent manner. ${ }^{12}$ Gd(III)-doped CQDs (GdCQDs) have been prepared. The Gd-CQDs displayed strong

$\dagger$ Electronic supplementary information (ESI) available. See DOI 10.1039/c7ra09785a 


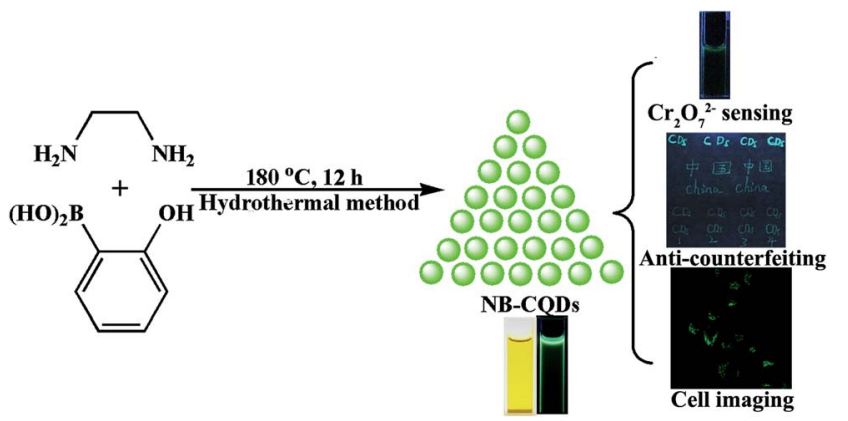

Fig. 1 Schematic illustration for the synthesis of NB-CQDs and the applications of NB-CQDs for sensing $\mathrm{Cr}_{2} \mathrm{O}_{7}{ }^{2-}$, anti-counterfeiting and cell imaging.

fluorescence, strong $\mathrm{T}_{1}$-weighted MRI contrast and low cytotoxicity, which indicated that the Gd-CQDs showed potential applications in biomedical fields. ${ }^{13}$ We have prepared nitrogen and phosphorus-doped carbon quantum dots (NP-CQDs) via one pot hydrothermal method. The NP-CQDs have been utilized to detect $\mathrm{Fe}^{3+}$ in aqueous solution with good sensitivity. ${ }^{\mathbf{1 4}}$ Liu and co-workers have synthesized boron and nitrogen-doped carbon quantum dots (NB-CQDs) from the hydrothermal treatment of branched polyethylenimine and 4-formylphenylboronic acid. The NB-CQDs exhibited excitation-dependent emission behavior and high quantum yield. And the as-prepared NB-CQDs have been applied for detecting $\mathrm{Fe}^{3+}$ and cell imaging. However, the NB-CQDs showed blue emission, which is not well suitable for the biological applications due to the bad tissue-penetrating ability of blue fluorescence. ${ }^{15}$ Thus, development of various CQDs with long-wavelength fluorescence emission is highly desirable.

Herein, we present a facile method for the synthesis of nitrogen and boron doped carbon quantum dots (NB-CQDs) with yellow-green emission through the hydrothermal method using 2-hydroxyphenylboronic acid and ethylenediamine as the precursors. The as-prepared NB-CQDs displayed bright yellowgreen emission, excellent stability in aqueous solution with high ionic strength and good biocompatibility. We found that the NB-CQDs could be employed to detect $\mathrm{Cr}_{2} \mathrm{O}_{7}{ }^{2-}$ in aqueous solution owing to the $\mathrm{Cr}_{2} \mathrm{O}_{7}{ }^{2-}$-caused fluorescence quenching. In the sensing system, as low as $0.5 \mu \mathrm{M} \mathrm{Cr}_{2} \mathrm{O}_{7}{ }^{2-}$ could be successfully detected and a good linear relationship in the range of $0-250 \mu \mathrm{M}$ has been obtained. And the NB-CQDs could be also used as ink for writing on different paper, revealing the NB-CQDs can be applied for anti-counterfeiting. Moreover, the NB-CQDs exhibited negligible cytotoxicity and good biocompatibility, which is favorable for bioimaging applications. And the NB-CQDs were successfully applied for fluorescence imaging in HeLa cells (Fig. 1).

\section{Experimental section}

\subsection{Materials}

Ethylenediamine (EDA) was bought from Energy Chemical Company (Shanghai, China). 2-Hydroxyphenylboronic acid (2-
HPBA), polyvinyl alcohol (PVA) and $\mathrm{K}_{2} \mathrm{Cr}_{2} \mathrm{O}_{7}$ were obtained from Aladdin Industrial Inc. (Shanghai, China). Other reagents were all of analytical reagent grade and used as received without further purification. Double-distilled water was used during the experiment.

\subsection{Characterization}

The excitation and emission spectra of NB-CQDs were recorded on a Cary Eclipse luminescence spectrometer (Varian, USA). Ultraviolet-visible (UV-vis) absorption spectra were measured at room temperature on a Lambda 650S UV-vis spectrometer (PerkinElmer, USA). Fourier transform-infrared (FT-IR) spectra were obtained using a Nicolet 5700 FT-IR spectrometer (ThermoElectron Corp., USA) with the $\mathrm{KBr}$ pellet technique in the range of 400-4000 $\mathrm{cm}^{-1}$. X-ray photoelectron spectroscope (XPS) spectra were carried on an X-ray photoelectron spectrometer (ESCALAB 250Xi, Thermo Scientific). Transmission electron microscopy (TEM) images were performed on a JEM-2100 transmission electron microscope operated at an acceleration voltage of $200 \mathrm{kV}$. Fluorescence lifetime and quantum yield were measured using a FLS 980 spectrofluorimeter (Edinburgh Instrument, UK). Photostability tests were conducted at room temperature using the light of $365 \mathrm{~nm}$ from an ultraviolet lamp (16W, CBIO-UV3A, Beijing CBIO Bioscience Technology Co. Ltd., Beijing, China). And photographs were gained using a Canon 700D digital camera.

\subsection{Synthesis of NB-CQDs}

In a typical procedure, 2-HPBA (about $71 \mathrm{mg}$ ) was dissolved in water $(10 \mathrm{~mL})$. EDA $(70 \mathrm{~mL})$ was then added into the above solution and stirred for about $10 \mathrm{~min}$. The mixture was sealed and heated at $180{ }^{\circ} \mathrm{C}$ for about 12 hours (h). After cooling to room temperature, a yellow solution was obtained and dialyzed in a cellulose ester dialysis membrane with a molecular weight cut-off of $500 \mathrm{Da}$ against distilled water at room temperature for about 12 h. Finally, a clean and light yellow solution containing NB-CQDs was gained and stored at $4{ }^{\circ} \mathrm{C}$. The concentration of NB-CQDs is about $0.5 \mathrm{mg} \mathrm{mL} \mathrm{m}^{-1}$ by estimating the weight of dried NB-CQDs.

\subsection{Detection of $\mathrm{Cr}_{2} \mathrm{O}_{7}{ }^{2-}$ with NB-CQDs}

For the detection of $\mathrm{Cr}_{2} \mathrm{O}_{7}{ }^{2-}, 750 \mu \mathrm{L}$ phosphate buffer $(0.2 \mathrm{M}$, pH 8.0), NB-CQDs solution $(200 \mu \mathrm{L})$ and different concentration of $\mathrm{K}_{2} \mathrm{Cr}_{2} \mathrm{O}_{7}$ were mixed together. And water was added into the mixture to keep the total volume of $3.0 \mathrm{~mL}$. After incubation for about $10 \mathrm{~min}$ at room temperature, the fluorescence intensity of NB-CQDs was recorded on the fluorescence spectrometer.

\subsection{Detection of $\mathrm{Cr}_{2} \mathrm{O}_{7}{ }^{2-}$ in tap water with NB-CQDs}

Tap water and lake water were collected in our laboratory and our campus. The real water samples were filtered through a $0.22 \mu \mathrm{m}$ filter to remove any suspended particles in water. $60 \mu \mathrm{L}$ tap water, different concentration of $\mathrm{K}_{2} \mathrm{Cr}_{2} \mathrm{O}_{7}$ and $750 \mu \mathrm{L}$ phosphate buffer were mixed together. Then NB-CQDs $(200 \mu \mathrm{L})$ were added into the mixture and water was used to dilute the above mixture to final volume of $3.0 \mathrm{~mL}$. After incubation for 
about $10 \mathrm{~min}$ at room temperature, the fluorescence signal of NB-CQDs was recorded with the fluorescence spectrometer.

\subsection{Preparation of NB-CQDs/PVA film}

$10 \mathrm{~mL}$ polyvinyl alcohol (PVA, 5\% wt) aqueous solution was first prepared. And $0.5 \mathrm{~mL}$ NB-CQDs solution was added the PVA solution under magnetic stirring. The mixture was then poured into a plastic Petri dish and dried in an oven with $60{ }^{\circ} \mathrm{C}$. The film was peeled off from the Petri dish.

\subsection{Cell viability assay of NB-CQDs}

HeLa cells were maintained in Dulbecco's modified Eagles Medium (DMEM) supplemented with 10\% fetal bovine serum and $1 \%$ penicillin-streptomycin at $37{ }^{\circ} \mathrm{C}$ in a humidified atmosphere $5 \% \mathrm{CO}_{2}$. To determine the cytotoxicity, cells were plated in triplicate at a density of $10^{4}$ cells per well in 96-well microplates. After incubation for $12 \mathrm{~h}$, the NB-CQDs ranging from 5 to $250 \mu \mathrm{g} \mathrm{mL}{ }^{-1}$ were added into the cells, and further cultured for about $24 \mathrm{~h}$. The viability of HeLa cells in the culture environment of NB-CQDs was then analyzed using 3-(4,5dimethyl-2-thiazolyl)-2,5-diphenyl-2- $H$-tetrazolium bromide (MTT) cytotoxicity assays.

\subsection{Cell imaging with NB-CQDs}

HeLa cells were seeded in $35 \mathrm{~mm}$ glass-bottomed dishes at a density of $3 \times 10^{5}$ cells per dish in standard culture media. After overnight culture, cells were treated with $100 \mu \mathrm{g} \mathrm{mL} \mathrm{mB}^{-1} \mathrm{NB}$ CQDs for about $20 \mathrm{~min}$, and then fluorescence images were acquired by confocal microscopy (Leica SP8 Confocal Laser Scanning Microscope). The cells were excited at $405 \mathrm{~nm}$ with a laser and the emission was collected between 500 and $550 \mathrm{~nm}$.

\section{Results and discussions}

\subsection{Synthesis of NB-CQDs}

The NB-CQDs was synthesized from the hydrothermal treatment of 2-HBPA and EDA at $180^{\circ} \mathrm{C}$ for about $12 \mathrm{~h}$ (Fig. 1). We first studied the effect of the ratio of 2-HBPA and EDA on the fluorescence properties of NB-CQDs. The NB-CQDs prepared from 2-HPBA and EDA with $1: 1,2: 1$ and $1: 2$ exhibited similar fluorescence properties. These NB-CQDs showed the maximum excitation wavelength at $410 \mathrm{~nm}$ and the maximum emission wavelength centered at $515 \mathrm{~nm}$. And their corresponding UV-vis absorption spectra were also similar. The maximum absorption wavelength was located at $410 \mathrm{~nm}$ (Fig. S1-S3†). To obtain the best NB-CQDs prepared from the suitable ratio of 2-HBPA and EDA, $I_{515} / A_{410}$ was employed to compare their fluorescence properties, where $I_{515}$ was the fluorescence intensity of NB-CQDs at the maximum emission wavelength of $515 \mathrm{~nm}, A_{410}$ was the absorbance of NB-CQDs at $410 \mathrm{~nm}$. The $A_{410}$ standed for the concentration of NB-CQDs, because the concentration of NB-CQDs is directly proportional to the $A_{410}$ based on the Beer's law. The greater $I_{515} / A_{410}$ showed the stronger fluorescence intensity of NB-CQDs. As shown in Fig. S4, $\uparrow$ we could see that the $I_{515} / A_{410}$ of the NB-CQDs prepared from 2-HBPA and EDA with the ratio of $1: 2$ was the largest.
Moreover, the CQDs from 2-HBPA or EDA were also prepared under the same condition. The CQDs from 2-HBPA showed the maximum excitation wavelength at about $290 \mathrm{~nm}$ and the maximum emission wavelength at $400 \mathrm{~nm}$, indicating that the CQDs from 2-HBPA exhibited blue emission. And there is no obvious absorption peak at $410 \mathrm{~nm}$ in the UV-vis absorption spectrum (Fig. S5 $\dagger$ ). For the CQDs from EDA, the maximum excitation wavelength at about $320 \mathrm{~nm}$ and the maximum emission wavelength at $390 \mathrm{~nm}$ were observed, revealing that the CQDs from EDA showed violet emission (Fig. S6†). The observations were much different from the NB-CQDs from 2HBPA and EDA. Therefore, we chose the ratio of 2-HBPA and EDA with $1: 2$ for the synthesis of NB-CQDs.

\subsection{Optical properties of NB-CQDs}

The native NB-CQDs solution showed yellow in visible light. However, bright yellow-green fluorescence was observed under a $365 \mathrm{~nm}$ UV light (Fig. 2A), indicating the formation of NBCQDs. The NB-CQDs were purified through dialysis. The purified NB-CQDs showed three peaks centered at about $225 \mathrm{~nm}$, $270 \mathrm{~nm}$ and $410 \mathrm{~nm}$ in the excitation spectrum (Fig. 2A). The excitation spectrum of NB-CQDs was similar with the UV-vis absorption spectrum (Fig. $\mathrm{S} 7 \dagger$ ). We then studied the fluorescence emission behavior of NB-CQDs at different excitation wavelengths. The NB-CQDs exhibited dual emission behavior when the excitation wavelength was in the range of 300-350 nm. The two maximum emission wavelengths were $400 \mathrm{~nm}$ and $515 \mathrm{~nm}$, respectively. When the excitation wavelength shifted longer wavelength, the NB-CQDs showed single emission behavior and the emission peak was located at about $515 \mathrm{~nm}$. Surprisingly, the NB-CQDs exhibited excitation-independent emission behavior, and the maximum emission wavelength was about $515 \mathrm{~nm}$. When the excitation wavelength was about $410 \mathrm{~nm}$, the fluorescent intensity of NB-CQDs at $515 \mathrm{~nm}$ was the largest (Fig. 2B). We thus selected $410 \mathrm{~nm}$ as the maximum excitation wavelength in the following experiments. Moreover, the absolute quantum yield of the NB-CQDs was measured to be about $6.59 \%$.

\subsection{Characterization of NB-CQDs}

We tried to characterize the surface structure and composition of the NB-CQDs with different methods. In the FT-IR spectrum of the NB-CQDs, the peak at about $3300 \mathrm{~cm}^{-1}$ could be attributed to the stretching vibration of $\mathrm{O}-\mathrm{H}$ or $\mathrm{N}-\mathrm{H}$ groups. ${ }^{15}$ The peaks at $2940 \mathrm{~cm}^{-1}$ and $2820 \mathrm{~cm}^{-1}$ were assigned to the stretching and bending vibrations of $\mathrm{C}-\mathrm{H}$ groups. ${ }^{15}$ The peak at $1660 \mathrm{~cm}^{-1}$ resulted from the stretching vibration of $\mathrm{C}=\mathrm{O}$ groups. ${ }^{15}$ The bending vibrations of $\mathrm{N}-\mathrm{H}$ groups could be observed at $1520 \mathrm{~cm}^{-1}$. The weak peak at about $1458 \mathrm{~cm}^{-1}$ could be attributed to the stretching vibration of $\mathrm{C}-\mathrm{N}$ groups. ${ }^{15}$ And the sharp peaks at about $1343 \mathrm{~cm}^{-1}$ and $1090 \mathrm{~cm}^{-1}$ could be assigned to the stretching vibrations $\mathrm{B}-\mathrm{O}$ and $\mathrm{C}-\mathrm{B}$ groups (Fig. 3A). ${ }^{16}$ The surface composition and elemental analysis for the NB-CQDs were characterized by XPS. The XPS survey spectrum of the NB-CQDs revealed four peaks at about 190.8, 284.6, 398.8 , and $531.0 \mathrm{eV}$, which were ascribed to B 1s, C 1s, N 1s and 

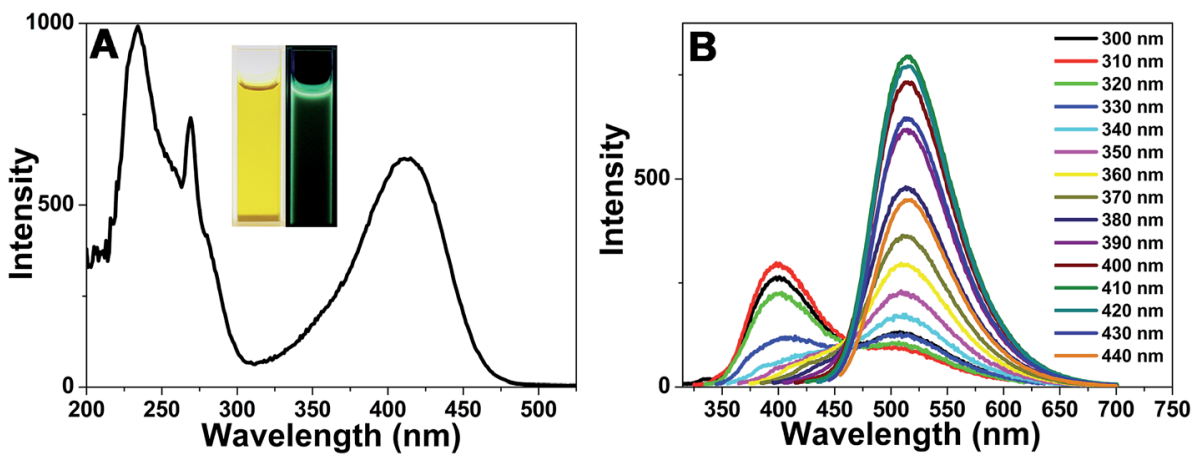

Fig. 2 (A) Excitation spectrum of NB-CQDs, inset: photographs of the native NB-CQDs in visible light and $365 \mathrm{~nm}$ UV light. (B) Emission spectra of NB-CQDs at different excitation wavelengths.
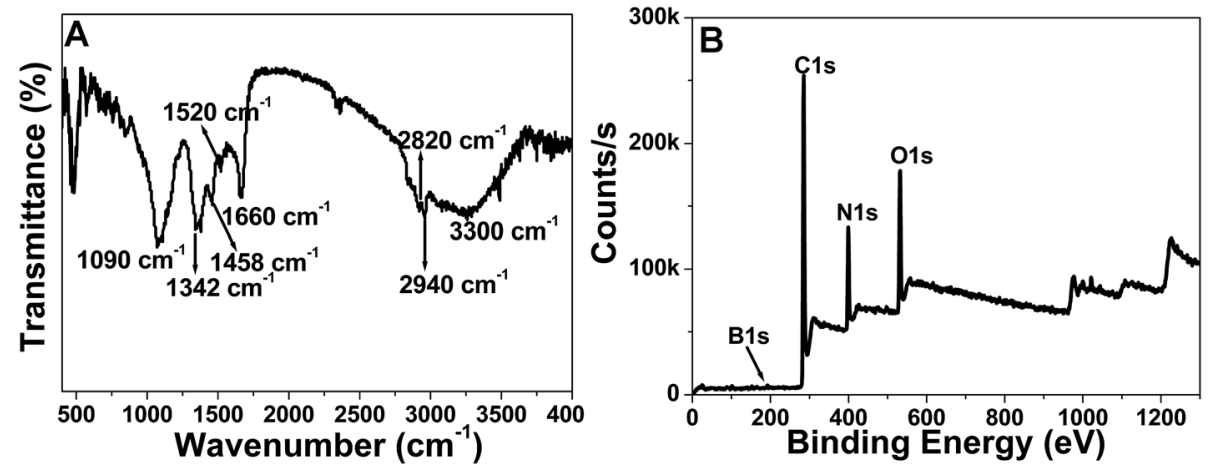

Fig. 3 (A) FT-IR spectrum of NB-CQDs. (B) XPS survey spectrum of NB-CQDs.

O 1s, respectively (Fig. 3B). In the high resolution C 1s spectrum, the C 1s peaks at 283.8, 284.5, 285.3, 287.6 and $290.2 \mathrm{eV}$ could be assigned to carbon in the form of $\mathrm{C}-\mathrm{B}, \mathrm{C}-\mathrm{C} / \mathrm{C}=\mathrm{C}, \mathrm{C}-\mathrm{O} /$ $\mathrm{C}-\mathrm{N}, \mathrm{C}=\mathrm{O}$ and $\mathrm{O}-\mathrm{C}=\mathrm{O}$, respectively (Fig. $\mathrm{S} 8 \mathrm{~A} \dagger)^{15-17}$ The $\mathrm{N} 1 \mathrm{~s}$ peaks at 398.5, 399.7 and $401.5 \mathrm{eV}$ showed that nitrogen existed mostly in the form of $\mathrm{N}-\mathrm{B}, \mathrm{N}-\mathrm{C}$ and $\mathrm{N}-\mathrm{H}$, respectively (Fig. S8B $\dagger$ ). ${ }^{18,19}$ The $\mathrm{O}$ 1s spectrum could be deconvoluted into two peaks at 530.5 and $531.8 \mathrm{eV}$, which were corresponding to $\mathrm{C}=\mathrm{O}$ and $\mathrm{C}-\mathrm{OH} / \mathrm{C}-\mathrm{O}-\mathrm{C}$, respectively (Fig. $\mathrm{S} 8 \mathrm{C} \dagger$ ). ${ }^{15}$ And the B $1 \mathrm{~s}$ spectrum could be separated into two peaks at 191.1 and $191.8 \mathrm{eV}$, indicating that the presence of $\mathrm{B}-\mathrm{N}$ and $\mathrm{B}-\mathrm{C}$
(Fig. S8D $\dagger$ ). ${ }^{16,18}$ These results were corresponding to the FT-IR results. And the above results also verified the as-prepared CQDs were nitrogen and boron-doped CQDs. The morphology and particle size of NB-CQDs were investigated by TEM. The NBCQDs were well-dispersed and nearly spherical, and the diameter was about $2.32 \mathrm{~nm}$ in average (Fig. 4).

\subsection{Stability of NB-CQDs}

The stability of NB-CQDs is of great importance for the wide applications of NB-CQDs. We first studied the effect of ionic
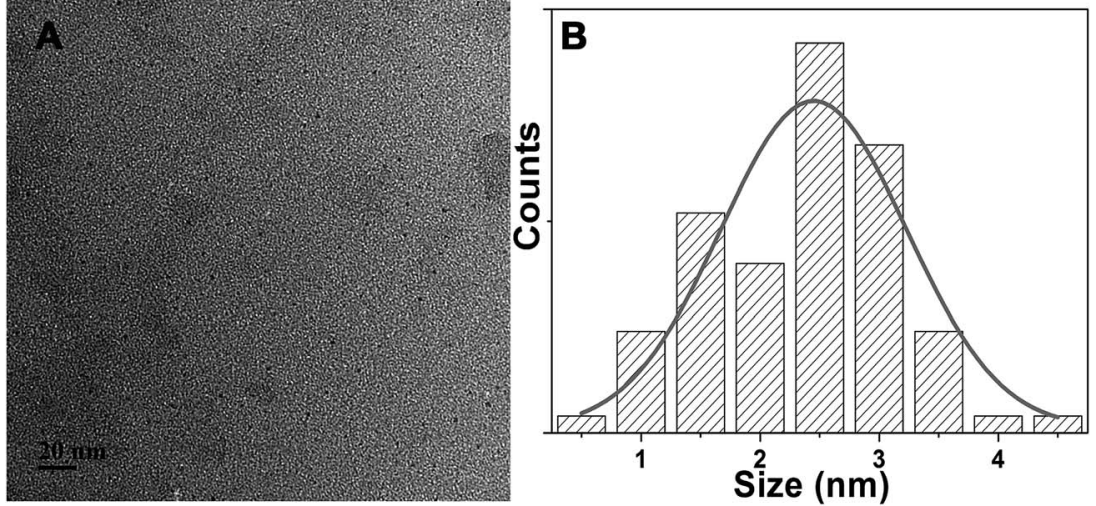

Fig. 4 (A) TEM image of NB-CQDs. (B) Size distribution of NB-CQDs. 
strength on the fluorescent intensity of NB-CQDs in the presence of different concentrations of NaCl. When the concentration of $\mathrm{NaCl}$ was as high as $1.2 \mathrm{M}$, the fluorescent intensity of NB-CQDs was little affected, revealing the excellent stability of the NB-CQDs in high-salt conditions (Fig. 5A). The photostability of NB-CQDs was also tested through the continuous irradiation of $365 \mathrm{~nm}$ UV light. The fluorescent intensity decreased slightly with the increase of irradiation time, and only $17 \%$ decrease in fluorescent intensity was observed after continuous irradiation for $30 \mathrm{~min}$ (Fig. 5B). The influence of scanning times on the fluorescent intensity was also investigated. The fluorescent intensity of NB-CQDs remained unchanged after scanning for 30 times (Fig. S9†). We also studied the temperature effect on the fluorescent intensity of NB-CQDs. Surprisingly, we noticed that the increase in temperature could result in a red shift of the fluorescence emission spectrum and a slight reduction in fluorescence intensity (Fig. S10†), revealing that the NB-CQDs may be constructed into nano-thermometers. These results revealed that the good stability of NB-CQDs. Moreover, the $\mathrm{pH}$ effect on the fluorescent intensity of NB-CQDs was also measured. The pH of the solution exhibited great influence on the fluorescent intensity of NB-CQDs. In the $\mathrm{pH}$ range of 1-8, the fluorescence intensity increased with the increase in the $\mathrm{pH}$ value, and the fluorescence intensity was the highest at $\mathrm{pH}$ 8. Further increase of the $\mathrm{pH}$ value could result in a gradual decrease in fluorescence intensity (Fig. 6A). The pH-responsive properties showed NB-CQDs the potential application in $\mathrm{pH}$ sensing. We thus chose $\mathrm{pH} 8.0$ for the following experiments.

\subsection{Effect of metal ions on the fluorescence intensity of NB- CQDS}

Due to the presence of rich functional groups on the surface of NB-CQDs, metal ions may greatly affect the fluorescence intensity of the NB-CQDs. For the test, 14 metal ions, including $\mathrm{Al}^{3+}, \mathrm{Ba}^{2+}, \mathrm{Ca}^{2+}, \mathrm{Cd}^{2+}, \mathrm{Co}^{2+}, \mathrm{Cr}^{3+}, \mathrm{Cu}^{2+}, \mathrm{Fe}^{3+}, \mathrm{Hg}^{2+}, \mathrm{Mg}^{2+}, \mathrm{Mn}^{2+}$, $\mathrm{Pb}^{2+}, \mathrm{Zn}^{2+}$, and $\mathrm{Cr}_{2} \mathrm{O}_{7}{ }^{2-}$, were chosen to study the influence on the fluorescence intensity of the NB-CQDs at $515 \mathrm{~nm}$. Their concentrations were $200.0 \mu \mathrm{M}$. As shown in Fig. 6B, only $\mathrm{Cr}_{2} \mathrm{O}_{7}{ }^{2-}$ could cause the significant decrease of the fluorescence intensity, and other metal ions showed slight impact on the fluorescence intensity of NB-CQDs. The results demonstrated that the as-prepared NB-CQDs could be utilized for sensing $\mathrm{Cr}_{2} \mathrm{O}_{7}{ }^{2-}$ in aqueous solution.

\subsection{Detection of $\mathrm{Cr}_{2} \mathrm{O}_{7}{ }^{2-}$ with NB-CQDs}

$\mathrm{Cr}(\mathrm{vI})$ is highly toxic and carcinogenic to humans, and cause different disorders and severe environmental problems. ${ }^{20}$ It is thus essential to develop a new method for selectively determination trace levels of $\mathrm{Cr}(\mathrm{vI}) . \mathrm{Cr}_{2} \mathrm{O}_{7}{ }^{2-}$ is the dominant form of $\mathrm{Cr}(\mathrm{vI})$ in nature. Thus, the detection of $\mathrm{Cr}_{2} \mathrm{O}_{7}{ }^{2-}$ is of great
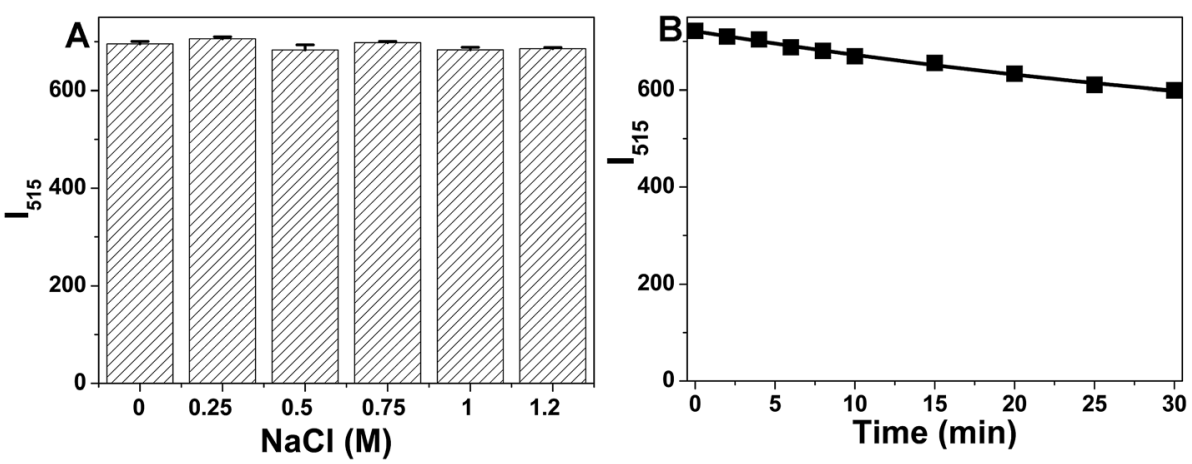

Fig. 5 (A) Effect of ionic strength on the fluorescent intensity of NB-CQDs. (B) Effect of irradiation time of $365 \mathrm{~nm} U \mathrm{~V}$ light of on the fluorescent intensity of NB-CQDs (performed in $\mathrm{pH} 8.0,50 \mathrm{mM}$ phosphate buffer; excitation wavelength is $410 \mathrm{~nm}$ ).
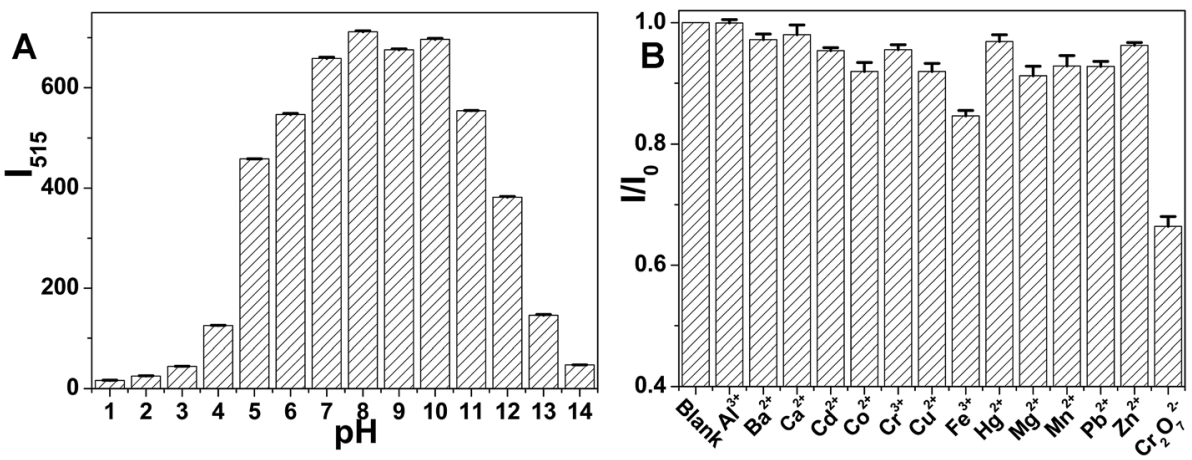

Fig. 6 (A) Effect of $\mathrm{pH}$ values on the fluorescent intensity of NB-CQDs. (B) Effect of various metal ions (200 $\mu \mathrm{M})$ on the fluorescent intensity of NB-CQDs (performed in $\mathrm{pH} 8.0,50 \mathrm{mM}$ phosphate buffer; excitation wavelength is $410 \mathrm{~nm}$ ). 
importance. The fluorescent detection of $\mathrm{Cr}_{2} \mathrm{O}_{7}{ }^{2-}$ could be realized by using NB-CQDs. Different concentration of $\mathrm{Cr}_{2} \mathrm{O}_{7}{ }^{2-}$ was added into the NB-CQDs solution, and the corresponding fluorescent spectra were recorded. With the increase of $\mathrm{Cr}_{2} \mathrm{O}_{7}{ }^{2-}$ concentration, the fluorescence intensity of NB-CQDs continuously decreased. And the shape and position of the fluorescence spectra did not changed obviously (Fig. 7A). A plot about the fluorescence intensity ratio $\left(I / I_{0}\right)$ against $\mathrm{Cr}_{2} \mathrm{O}_{7}{ }^{2-}$ was obtained. Notably, a good linearity $(R=0.995)$ between $I / I_{0}$ and the concentration of $\mathrm{Cr}_{2} \mathrm{O}_{7}{ }^{2-}$ in the range of 0-250 $\mu \mathrm{M}$ was established, revealing that the as-prepared NBCQDs have the potential to detect $\mathrm{Cr}_{2} \mathrm{O}_{7}{ }^{2-}$ in aqueous solution (Fig. 7B). Moreover, the lowest detection concentration of $\mathrm{Cr}_{2} \mathrm{O}_{7}{ }^{2-}$ was $0.5 \mu \mathrm{M}$, which was not comparable to that of the reported nanosensors for $\mathrm{Cr}_{2} \mathrm{O}_{7}{ }^{2-}$ (Table $\left.\mathrm{S} 1 \dagger\right) .^{20-24}$ However, the value was much below the allowed lowest concentration in drinking water set by the US Environmental Protection Agency. ${ }^{21,22}$ Most importantly, the linear range is much wider than those reported results (Table $\mathrm{S} 1 \dagger$ ), revealing that the NBCQDs have the potential to detect $\mathrm{Cr}_{2} \mathrm{O}_{7}{ }^{2-}$ in some practical samples.

\subsection{Practical application of NB-CQDs for the detection of $\mathrm{Cr}_{2} \mathrm{O}_{7}{ }^{2-}$ in real water samples}

The applicability of the NB-CQDs-based fluorescent sensor was validated by the detection of $\mathrm{Cr}_{2} \mathrm{O}_{7}{ }^{2-}$ in tap water and lake water. The real water samples were diluted to 50 times. The analysis results were shown in Table $\mathrm{S} 2 . \dagger \mathrm{Cr}_{2} \mathrm{O}_{7}{ }^{2-}$ was not determined in tap water and lake water, indicating that there was no $\mathrm{Cr}_{2} \mathrm{O}_{7}{ }^{2-}$ or the $\mathrm{Cr}_{2} \mathrm{O}_{7}{ }^{2-}$ concentration in the two real water samples was lower than the lowest detection concentration of NB-CQDs. Then, different concentration of $\mathrm{Cr}_{2} \mathrm{O}_{7}{ }^{2-}$ was added into the NB-CQDs solution and the corresponding fluorescent intensity was recorded. Compared with the initial addition of $\mathrm{Cr}_{2} \mathrm{O}_{7}{ }^{2-}$, the final recoveries were determined between $102.0 \%$ and $113.4 \%$, revealing that the NB-CQDs could be applied to detect $\mathrm{Cr}_{2} \mathrm{O}_{7}{ }^{2-}$ in some real water samples.

\subsection{Detection mechanism of $\mathrm{Cr}_{2} \mathrm{O}_{7}{ }^{2-}$ with NB-CQDS}

The fluorescence quenching mechanism of NB-CQDs against $\mathrm{Cr}_{2} \mathrm{O}_{7}{ }^{2-}$ is still a matter of current debate and requires further elucidation. The UV-vis absorption spectra were first utilized to elaborate the fluorescence quenching mechanism of NB-CQDs. As indicated in Fig. S11, $\dagger$ the maximum absorption spectrum of $\mathrm{K}_{2} \mathrm{Cr}_{2} \mathrm{O}_{7}$ partially overlapped with the excitation spectrum of NB-CQDs, suggesting that the $\mathrm{Cr}_{2} \mathrm{O}_{7}{ }^{2-}$-caused fluorescence quenching of NB-CQDs was ascribed to the inner filter effect. ${ }^{19}$ Moreover, the addition of NB-CQDs could enhance the absorbance of $\mathrm{K}_{2} \mathrm{Cr}_{2} \mathrm{O}_{7}$ at $370 \mathrm{~nm}$. And the addition of $\mathrm{K}_{2} \mathrm{Cr}_{2} \mathrm{O}_{7}$ could change the absorption spectrum of NB-CQDs, demonstrating that the fluorescence quenching mechanism was static quenching mechanism (Fig. S12 $\dagger$ ). ${ }^{25,26}$ And the fluorescence lifetime test can be utilized to reveal the fluorescence quenching mechanism because the static quenching process is related

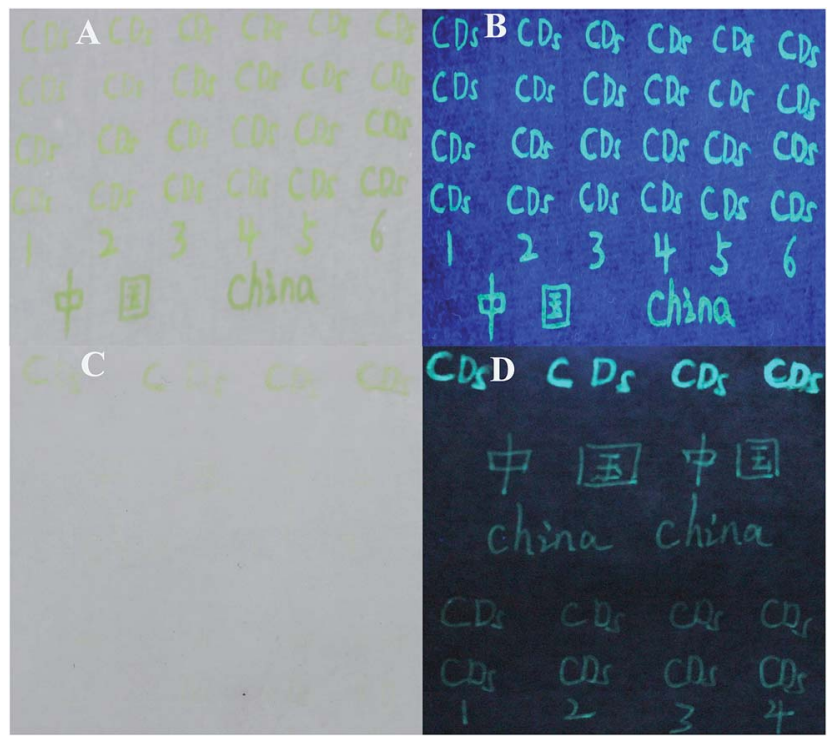

Fig. 8 Handwritten letters, number and Chinese characters using NBCQDs as ink on Xuan paper ( $A$ and $B$ ) and weighing paper ( $C$ and $D$ ) under visible light ( $A$ and $C$ ) and $365 \mathrm{~nm}$ UV light ( $B$ and $D$ ).
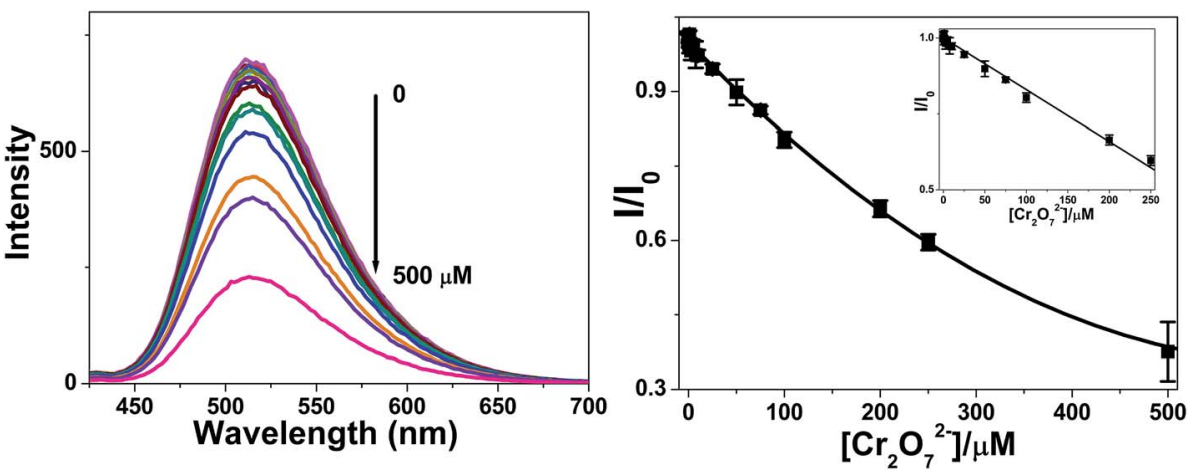

Fig. 7 (A) Fluorescence emission spectra of NB-CQDs in the presence of different concentration of $\mathrm{Cr}_{2} \mathrm{O}_{7}{ }^{2-}$. (B) $\mathrm{The}$ plot of $/ / \mathrm{I}_{0}$ versus of the concentration of $\mathrm{Cr}_{2} \mathrm{O}_{7}{ }^{2-}$, inset: the linear relationship between $/ / l_{0}$ and the concentration of $\mathrm{Cr}_{2} \mathrm{O}_{7}{ }^{2-}$ in the range from 0 to $250 \mu \mathrm{M}$ (performed in $\mathrm{pH}$ 8.0, $50 \mathrm{mM}$ phosphate buffer; excitation wavelength is $410 \mathrm{~nm}, /$ and $I_{0}$ were the fluorescence intensity of NB-CQDs in the presence and absence of $\mathrm{Cr}_{2} \mathrm{O}_{7}{ }^{2-}$, respectively). 

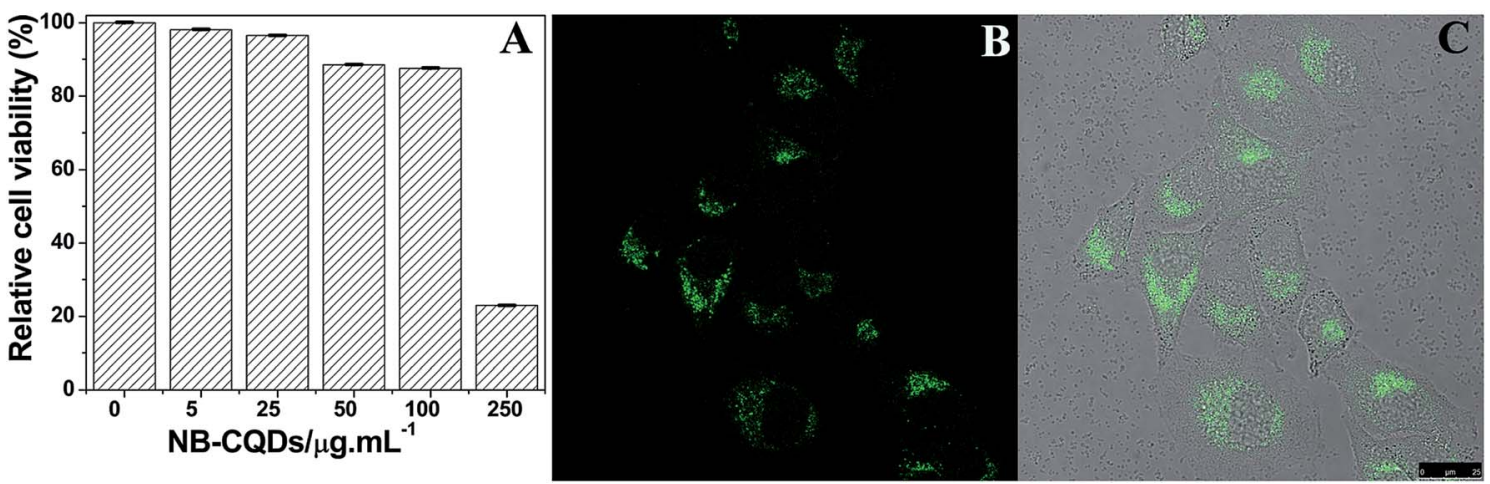

Fig. 9 (A) Cell viability of HeLa cells after $24 \mathrm{~h}$ incubated with NB-CQDs by MTT assay. (B) Confocal fluorescence images of HeLa cells labeled with NB-CQDs $\left(100 \mu \mathrm{g} \mathrm{mL}^{-1}\right)$ excited with a $405 \mathrm{~nm}$ laser and bright field (C), the scale bar is $25 \mu \mathrm{m}$.

to the unchanged fluorescence lifetime of NB-CQDs. ${ }^{25,26}$ The lifetime of NB-CQDs was about 3.3581 ns. However, in the presence of 100 and $500 \mu \mathrm{M}$, the lifetime of NB-CQDs was $3.3857 \mathrm{~ns}$ and $3.3708 \mathrm{~ns}$, respectively (Fig. S13†). The altered lifetime indicated the fluorescence quenching mechanism of NB-CQDs by $\mathrm{Cr}_{2} \mathrm{O}_{7}{ }^{2-}$ was dynamic quenching. ${ }^{25}$ These results could effectively confirm the inner filter effect contributed the fluorescence quenching of NB-CQDs. And static and dynamic quenching also caused the fluorescence quenching mechanism.

\subsection{Anti-counterfeiting of NB-CQDs}

We further investigated the application of NB-CQDs for anticounterfeiting. We first found that the NB-CQDs could be used as ink for writing letters, number and Chinese characters Xuan Paper and weighing paper. With the decrease of the concentration of NB-CQDs, these letters, number and Chinese characters gradually changed from light yellow to colorless under visible light. However, upon the irradiation of $365 \mathrm{~nm} \mathrm{UV}$ light, they were clearly observed with blue color (Fig. 8). And the blue fluorescence still retained after three months, revealing that the NB-CQDs could be utilized for anti-counterfeiting. The NB-CQDs could be mixed with PVA solution to fabricate NBCQDs/PVA film, which was transparent and exhibited light yellow under visible light. However, the film showed blue emission in $365 \mathrm{~nm} \mathrm{UV}$ light (Fig. S14†). In the excitation spectrum of NB-CQDs/PVA film, the maximum excitation wavelength was located at about $420 \mathrm{~nm}$, which showed a weak red shift when compared with the NB-CQDs in solution (Fig. S15A $\dagger$ ). And the emission spectra of NB-CQDs/PVA film excited with different wavelengths were shown in Fig. S15B. $\dagger$ The NB-CQDs/PVA film displayed wavelength-independent emission and the maximum fluorescence emission at about $450 \mathrm{~nm}$ with excitation wavelength of $420 \mathrm{~nm}$. Surprisingly, the maximum fluorescence emission wavelength exhibited a large blue shift when compared with NB-CQDs in aqueous solution, which may be attributed to the alteration of average NB-CQDs $\cdots$ NB-CQDs distance. ${ }^{27,28}$ The compactness of the NB-CQDs/PVA film induced the interaction between these NB-CQDs, thus increasing the average NB-CQDs $\cdots$ NB-CQDs distance, resulting into the blue shift in the fluorescence emission. ${ }^{28}$

\subsection{Cell imaging with NB-CQDs}

In order to evaluate the cytotoxicity of the as-prepared NB-CQDs, MTT assays were carried out. ${ }^{19,29,30}$ Therefore, HeLa cells were selected as representative cell lines to incubate with NB-CQDs at different concentrations for $24 \mathrm{~h}$. As shown in Fig. 9A, the cell viability decreased by $17 \%$ when the concentration of N-CQDs reached up to $100 \mu \mathrm{g} \mathrm{mL} \mathrm{m}^{-1}$. These results clearly revealed that the as-synthesized NB-CQDs showed low cytotoxicity to living cells when compared with those semiconductor QDs. ${ }^{31}$ The good water solubility and low cytotoxicity of the NB-CQDs could be applied for cell imaging with confocal microscopy. And HeLa cells were incubated with NB-CQDs $\left(100 \mu \mathrm{g} \mathrm{mL}{ }^{-1}\right)$ for $20 \mathrm{~min}$ at $37^{\circ} \mathrm{C}$. As shown in Fig. 9B and C, a bright blue-green fluorescence was observed inside the cells, indicating that the NB-CQDs were quickly internalized by the HeLa cells. The above results suggested that the NB-CQDs exhibited good biocompatibility, low cell cytotoxicity, and could be used for cell imaging.

\section{Conclusions}

In summary, we have presented a method for the synthesis of NB-CQDs through the hydrothermal method using 2-HPBA and EDA in aqueous solution. The as-prepared NB-CQDs exhibited bright yellow-green emission, excellent stability in aqueous solution with high ionic strength and good biocompatibility. The NB-CQDs could be utilized to sense $\mathrm{Cr}_{2} \mathrm{O}_{7}{ }^{2-}$ in aqueous solution due to the $\mathrm{Cr}_{2} \mathrm{O}_{7}{ }^{2-}$-induced fluorescence quenching. In the sensing system, as low as $0.5 \mu \mathrm{M} \mathrm{Cr}_{2} \mathrm{O}_{7}{ }^{2-}$ can be successfully detected and a good linear relationship in the range of $0-250 \mu \mathrm{M}$ has been obtained. And the NB-CQDs can be also used as ink for writing on different paper, revealing the NB-CQDs can be applied for anti-counterfeiting. Moreover, the NB-CQDs exhibited low cytotoxicity and good biocompatibility, which is favorable for bioimaging applications. And the NB-CQDs were successfully applied for fluorescence imaging in HeLa cells. Therefore, the NB-CQDs exhibit great potential applications in various fields.

\section{Conflicts of interest}

There are no conflicts to declare. 


\section{Acknowledgements}

The work was financially supported by the National Natural Science Foundation of China (21401113, 21575032), the Natural Science Foundation of Henan Department of Science \& Technology (172102310668), the Natural Science Foundation of Nanyang Normal University (ZX2014043), scientific research and service platform fund of Henan Province (2016151); the fund scientific and technological innovation team of water ecological security for Water Source Region of Mid-line of South-to-North Diversion Project of Henan Province (17454), the Fundamental Research Funds for the Central Universities (PYBZ1707, buctrc201607), and Open Ground from State Key Laboratory of Chemo/Biosensing and Chemometrics Hunan University.

\section{Notes and references}

1 L. Yan, Y. Zhang, B. Xu and W. Tian, Nanoscale, 2016, 8, 2471-2487.

2 A.-J. Wang, H. Li, H. Huang, Z.-S. Qian and J.-J. Feng, J. Mater. Chem. C, 2016, 4, 8146-8160.

3 Y. Guo, F. Cao, X. Lei, L. Mang, S. Cheng and J. Song, Nanoscale, 2016, 8, 4852-4863.

4 Y. Guo, L. Zhang, S. Zhang, Y. Yang, X. Chen and M. Zhang, Biosens. Bioelectron., 2015, 63, 61-71.

5 S. M. Ng, M. Koneswaran and R. Narayanaswamy, RSC Adv., 2016, 6, 21624-21661.

6 H.-B. Wang, Y. Chen, Y. Li, H.-D. Zhang and J.-T. Cao, RSC Adv., 2015, 5, 94099-94104.

7 H. Yu, R. Shi, Y. Zhao, G. I. N. Waterhouse, L.-Z. Wu, C.-H. Tung and T. Zhang, Adv. Mater., 2016, 28, 9454.

8 Z. Zhang, T. Zheng, X. Li, J. Xu and H. Zeng, Part. Part. Syst. Charact., 2016, 33, 457-472.

9 Q. Zhang, C.-F. Wang, L.-T. Ling and S. Chen, J. Mater. Chem. C, 2014, 2, 4358-4373.

10 Y. H. Yuan, Z. X. Liu, R. S. Li, H. Y. Zou, M. Lin, H. Liu and C. Z. Huang, Nanoscale, 2016, 8, 6770-6776.

11 Z. Ma, H. Ming, H. Huang, Y. Liu and Z. Kang, New J. Chem., 2012, 36, 861-864.

12 S. Lu, S. Guo, P. Xu, X. Li, Y. Zhao, W. Gu and M. Xue, Int. J. Nanomed., 2016, 11, 6325-6336.

13 A. B. Bourlinos, A. Bakandritsos, A. Kouloumpis, D. Gournis, M. Krysmann, E. P. Giannelis, K. Polakova, K. Safarova,
K. Hola and R. Zboril, J. Mater. Chem., 2012, 22, 2332723330.

14 Y. Guo, F. Cao and Y. Li, Sens. Actuators, B, 2017, DOI: 10.1016/j.snb.2017.1008.1104.

15 J. Liu, J. Li, L. Xu, Y. Qiao and J. Chen, Ind. Eng. Chem. Res., 2017, 56, 3905-3912.

16 P. Shen and Y. Xia, Anal. Chem., 2014, 86, 5323-5329.

17 G. Caballero-Manrique, E. Brillas, F. Centellas, J. A. Garrido, R. M. Rodriguez and P.-L. Cabot, Catalysts, 2015, 5, 815-837.

18 D. He, W. Cheng, J. Qin, J. Yue, E. Xie and G. Chen, Appl. Surf. Sci., 2002, 191, 338-343.

19 G. Li, H. Fu, X. Chen, P. Gong, G. Chen, L. Xia, H. Wang, J. You and Y. Wu, Anal. Chem., 2016, 88, 2720-2726.

20 X. Liu, T. Li, Q. Wu, X. Yan, C. Wu, X. Chen and G. Zhang, Talanta, 2017, 165, 216-222.

21 W. Chen, F. Cao, W. Zheng, Y. Tian, Y. Xianyu, P. Xu, W. Zhang, Z. Wang, K. Deng and X. Jiang, Nanoscale, 2015, 7, 2042-2049.

22 C. Dong, G. Wu, Z. Wang, W. Ren, Y. Zhang, Z. Shen, T. Li and A. Wu, Dalton Trans., 2016, 45, 8347-8354.

23 M. Cui, G. Song, C. Wang and Q. Song, Microchim. Acta, 2015, 182, 1371-1377.

24 L. Zhu, X. Peng, H. Li, Y. Zhang and S. Yao, Sens. Actuators, B, 2017, 238, 196-203.

25 Y. Guo, L. Zhang, F. Cao and Y. Leng, Sci. Rep., 2016, 6, 35795.

26 Y. Guo, L. Zhang, F. Cao, L. Mang, X. Lei, S. Cheng and J. Song, Anal. Methods, 2016, 8, 2723-2728.

27 Q. Benito, X. F. Le Goff, S. Maron, A. Fargues, A. Garcia, C. Martineau, F. Taulelle, S. Kahlal, T. Gacoin, J.-P. Boilot and S. Perruchas, J. Am. Chem. Soc., 2014, 136, 11311-11320.

28 Z. Wu, J. Liu, Y. Gao, H. Liu, T. Li, H. Zou, Z. Wang, K. Zhang, Y. Wang, H. Zhang and B. Yang, J. Am. Chem. Soc., 2015, 137, 12906-12913.

29 S. Zhu, Q. Meng, L. Wang, J. Zhang, Y. Song, H. Jin, K. Zhang, H. Sun, H. Wang and B. Yang, Angew. Chem., Int. Ed., 2013, 52, 3953-3957.

30 R. Atchudan, T. N. J. I. Edison and Y. R. Lee, J. Colloid Interface Sci., 2016, 482, 8-18.

31 S. Ghaderi, B. Ramesh and A. M. Seifalian, J. Drug Targeting, 2011, 19, 475-486. 